\title{
An Adaptive Resource Allocation Scheme of the Forward Channel in CDMA System
}

\author{
Sung-Hong Wie and Dong-Ho Cho \\ Communication and Information System Laboratory \\ Department of Electrical Engineering \\ Korea Advanced Institute of Science and Technology \\ 373-1 Kusong-Dong, Yusong-Gu, Taejon, 305-701 Korea \\ (TEL: +82-42-869-3467, FAX: +82-42-867-0550, E-mail: dhcho@ee.kaist.ac.kr)
}

\begin{abstract}
In this paper, we consider not only the position of a mobile station but also the system load for adaptive resource allocation suitable to the broadband service. When a mobile station is near the cell boundary, the proposed scheme restricts the resource assigned to that mobile. But when the system load is low, our scheme relaxes the restriction of resource for mobiles. This scheme increases the channel utilization, the system capacity and the flexibility of the resource management.
\end{abstract}

Keywords—Resource Allocation, multimedia communications, CDMA

\section{INTRODUCTION}

Current CDMA(Code Division Multiple Access) systems based on IS-95A support only a circuit-switched voice communication. But IMT-2000 such as cdma2000 and ETSI UTRA should support the broadband communication service([1]-[2]). To support the broadband service, a MAC(Medium Access Control) protocol must have a bandwidth allocation algorithm suitable to that service. IMT-2000 system should consider an asymmetric traffic property. A reverse channel in VOD or web service, conveys frequently request packets for an wanted service. Therefore the traffic amount of a reverse channel is small. On the other hand, the forward channel must transport larger information as an acknowledge of the request packet. There are two ways to transport a large information in the CDMA system. One way is MC(MultiCode)-CDMA scheme. That is, the assignment of multiple codes in the forward channel supports the broadband service. The other way is VG(Variable-Gain)-CDMA scheme. When the broadband service is needed, it reduces a processing gain and increases a transmission rate. Using these two methods, the forward channel can convey larger information. But when there are many users to demand the broadband service, a few users could be served. Thus the performance of the forward channel should be improved to accept these requests for the broadband communication service. And the resource allocation scheme of the forward channel should be made very efficiently and reasonably. In CDMA system, large resource allocations for some mobiles result in large transmission rate and large power. It means that other mobiles suffer the large interference. Ref. [3] and [4] propose the code assignment scheme based on LIDA(Load and Interference based Demand Assignment). But this scheme is applied only to the reverse channel. We apply it to the forward channel and extend this scheme to the resource allocation scheme of the forward channel.

We consider mainly MC-CDMA system. In this system, the resource means the number of codes. The our proposed scheme is divided into two parts. In the first part, the proposed scheme 
considers the position of a mobile station. Because the position of a mobile station is related with the inter-cell interference, many codes(large resource) are allocated to the mobile near the base station and the number of codes allocated to the mobile near the cell boundary is limited as small as possible. This code assignment scheme based on the mobile position reduces an unnecessary interference to other base stations. Because a CDMA system is the interference-limited system, the unnecessary interference reduction results in the increasement of system capacity. In the second part, the proposed scheme is based on the system load. When a mobile station is near the cell boundary, the proposed scheme based on the position of mobile station minimizes the number of codes assigned to that mobile. But this scheme may not be the best under the present system state. When the system load is low, it is unnecessary to limit the number of codes for mobiles. Thus, our proposed scheme has the resource allocation scheme based on the system load. This proposed scheme increases the channel utilization, the system capacity and the flexibility of the code management.

Following this introduction, we explain the adaptive resource allocation scheme in Section II. We also show the numerical results in Section III. Finally, conclusions and further study are described in Section IV.

\section{Adaptive Resource Allocation SCHEME}

In this section, we propose an adaptive resource allocation scheme of the forward channel. In Multi-Code CDMA system, the resource allocation scheme is equivalent to the code assignment scheme. As a matter of convenience, the target system is assumed to be the $\mathrm{MC}(\mathrm{Multi}$ Code)-CDMA system. The number of assigned codes is based on the states of a base station and a mobile station. The proposed scheme is

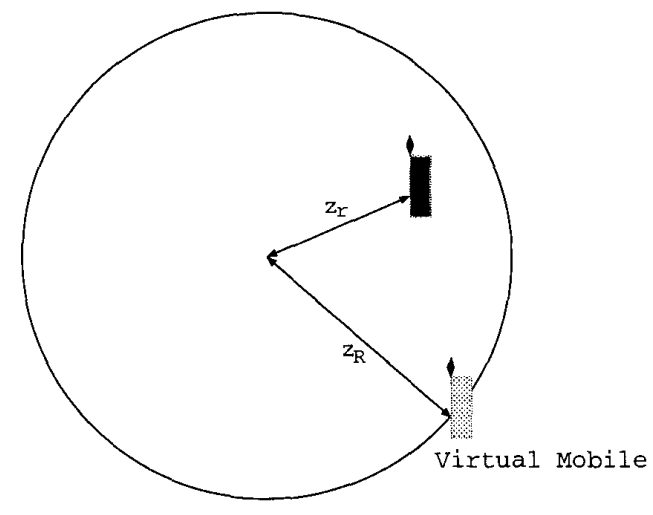

Fig. 1. System Model (Virtual Mobile)

divided into two parts which are explained in the subsection $\mathrm{A}$ and $\mathrm{B}$. The subsection $\mathrm{C}$ explains the scheme for VG-CDMA system.

\section{A. Resource Allocation based on Mobile Position}

To simplify the problem, we suppose the MCCDMA system with perfect power control. And it is assumed that forward channels are perfectly orthogonal. In these assumptions, forward channels are affected from only the inter-cell interference. Thus we consider the inter-cell interference for the code allocation and set a limitation to the number of allocated codes. To calculate the inter-cell interference, we introduce 'virtual mobile'. Virtual mobile is an imaginary mobile and is located on a boundary of the cell. Fig. 1 shows the system model and virtual mobile concept. Using a limitation of the interference to the virtual mobile, we restrict the resource to the mobile. We assume that the basic rate of MC-CDMA system is the same as the channel rate for a voice communications and the interference to a virtual mobile of a basic channel is one. That is, if a basic channel is established for the mobile on the cell boundary, the interference to a virtual mobile is one.

We define parameters as follows :

- $\alpha=$ the voice activity factor

- $r=$ the distance between a mobile and a cell boundary

- $R=$ the cell radius 
- $z_{r}=$ the path loss from host-cell to a mobile at distance $r$

- $m=$ the number of allocated codes to a mobile

- $M=$ the maximum number of codes allocated to a mobile

Through a limitation of the interference to the virtual mobile, we restrict the number of allocated codes to the mobile. The path loss $z_{r}$ can be calculated by using the pilot strength measurement and $z_{R}$ is the system parameter. When $m$ codes are allocated to the mobile at distance $r$, the interference to the virtual mobile is given by

$$
I_{v}=m\left(\frac{z_{r}}{z_{R}}\right)
$$

We suggest that $I_{v}$ should be less than the interference value of one voice channel. Thus Eq. (1) is restricted by

$$
I_{v}=m\left(\frac{z_{r}}{z_{R}}\right) \leq \alpha .
$$

Therefore, the number of codes is limited as follows :

$$
m \leq \min \left(M,\left(\frac{z_{R}}{z_{r}}\right) \alpha\right) .
$$

As seen by Eq. (3), a small number of codes is assigned to a mobile at long distance (large $z_{r}$ ). This scheme restricts the inter-cell interference and increases the system capacity.

\section{B. Resource Allocation based on System Load}

Previous section sets a limitation to the virtual mobile in view of the interference and the number of allocated codes. However because the number of allocated codes is determined by the mobile's position, this scheme may decrease the channel utilization. If neighbor cell's load is low, it is possible to allocate more codes to the mobile. That is, the number of allocated codes should be decided according to the system condition as well as mobile condition. Therefore, we introduce the variable factor $x$ into the right term of Eq. (2). The variable, $x$, is related to the system load.

$$
I_{v}=m\left(\frac{z_{r}}{z_{R}}\right) \leq \alpha x
$$

We define the system load parameter, $f$. The parameter, $f$, has a positive value and represents the system load for the forward channel.

$$
\begin{array}{r}
f \propto(\text { SystemLoad }) \\
0 \leq f \leq 1
\end{array}
$$

When the system load, $f$, is low, the maximum resource allocation is possible. That is, if $f$ is zero, $x$ is infinity. And similarly when $f$ is one, $x$ is zero. Many relationships between $f$ and $x$ is possible. We define the relationship as follows:

$$
x=A \ln (1 / f)
$$

where $A$ is a positive real number. To obtain the system load, we assume that neighbor BS(base station)s exchange periodically the load of the forward channel and calculate the system load, $f$. By introducing the system load, $f$, Eq. (2) could be modified as following

$$
I_{v}=m\left(\frac{z_{r}}{z_{R}}\right) \leq \alpha A \ln (1 / f) .
$$

And the number of codes is obtained by

$$
m \leq \min \left(M,\left(\frac{z_{R}}{z_{r}}\right) \alpha A \ln (1 / f)\right) .
$$

Eq. (8) shows that a large system load restricts the assigned number of codes. This scheme increases the channel utilization, the system capacity and the flexibility of the code management by considering the system load.

\section{Resource Allocation for VG(Variable-Gain)- CDMA}

To serve the broadband service, VG-CDMA system can be adopted. When the broadband service is needed, the system reduces a processing gain and enlarges a transmission rate. The large transmission rate results in a large transmission power(large interference). Although the 
physical properties of VG-CDMA are different from MC-CDMA, high level properties are the same. In VG-CDMA system, a variable $m$ means the ratio of the allowed rate over the basic rate. And $m$ may not be a integer but a real number. We define the rate set $S_{R}$ which contains the possible rates as follows:

$$
S_{R}=\left\{r_{1} / r_{1}, r_{2} / r_{1}, r_{3} / r_{1}, \cdots, r_{\max } / r_{1}\right\}
$$

where $r_{1}$ is a lowest rate and $r_{\max }$ is a maximum rate. Therefore, Eq. (8) should be changed to

$$
m \leq\left(\frac{z_{R}}{z_{r}}\right) \alpha A \ln (1 / f) \text { and } m \in S_{R}
$$

As seen by Eq. (10), this resource allocation scheme for VG-CDMA is the same as MCCDMA. Therefore, this scheme is easily applicable to the mixed system with MC-CDMA and VG-CDMA.

\section{Numerical Results}

In this section, numerical results for MCCDMA system are shown. To represent clearly the property of our scheme, we neglect the fading effect. Then, Eq. (7) and (8) are changed into functions of mobile's position. We suppose that a path loss exponent is 4 .

$$
\begin{array}{r}
I_{v}=m\left(\frac{r}{R}\right)^{4} \leq \alpha A \ln (1 / f) \\
m \leq \min \left(8,\left(\frac{R}{r}\right)^{4} \alpha A \ln (1 / f)\right) .
\end{array}
$$

We assume that the voice activity factor, $\alpha$, is 0.4 and a constant, $A$, is 10 . Fig. 2 shows the number of allocated codes according to the mobile's position and system load. This scheme allocates more codes when a mobile is closer to a base station. And as the system load is lower, more codes could be assigned to a mobile. When the load is 0.1 , the maximum codes could be allocated to the mobile on the cell boundary. However, when the load is 0.9 , none codes is assigned to the mobile on the cell boundary. This figure shows that our scheme would manages effectively

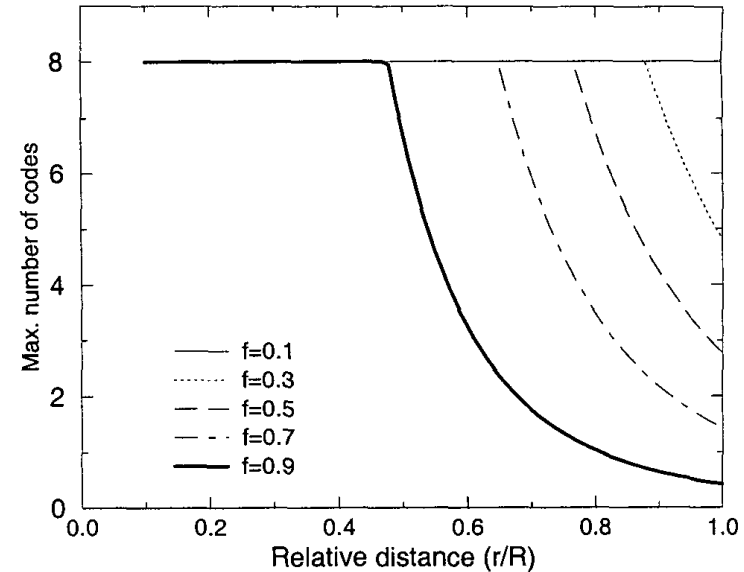

Fig. 2. Code assignment according to mobile's position and system load

the resource according to the mobile position and the system load. Fig. 3 shows that the proposed adaptive scheme controls the inter-cell interference according to the system load. The unrestricted code assignment scheme can not control the interference to neighbor cells. However, because our scheme limits the resource according to the mobile position and the system load, the interference to other cells is restricted below the certain threshold value. The threshold value is determined by the system load as seen by Eq. (11). This figure shows that our scheme reduces the inter-cell interference. That is, it prevents the fluctuation of the excessive transmitted power. The reduction of interference results in the capacity increasement and the efficient resource utilization.

\section{Conclusions and Further Study}

In this paper, we analyze the adaptive resource allocation scheme of the forward channel. This proposed scheme could be applied to Multi-Code CDMA systems and Variable-Gain CDMA systems. The numerical results show that our proposed scheme has adaptability to the position of the mobile and system load. Also our proposed scheme shows the inter-cell interference reduction to other cells. 


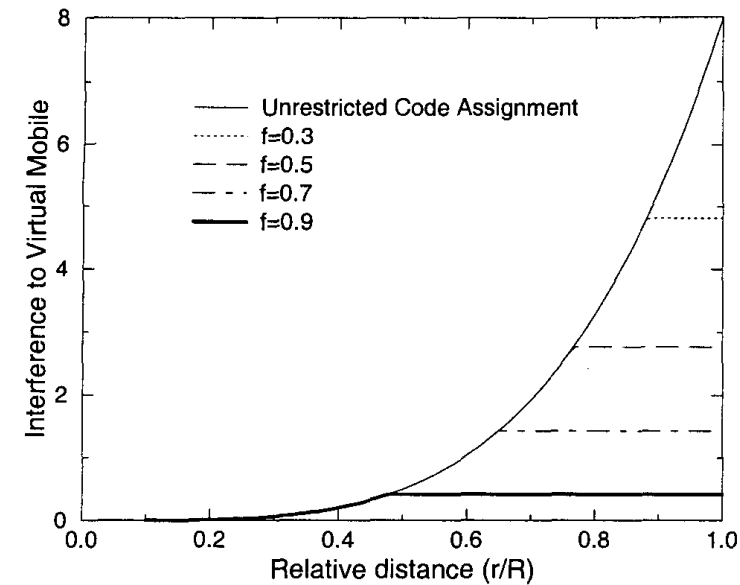

Fig. 3. Interference reduction by adaptive code assignment

There are some further problems. To obtain the system load, $f$, further studies are needed. In this paper, we assume that neighbor base stations exchange periodically the load of the forward channel. However, we need the research which mobiles measure the noise level and predict the load of forward channel. This research helps us to the distributed resource management scheme based on the smart antenna. Using the smart antenna, the cell's heavy traffic condition may not mean the large inter-cell interference. In this case, each mobile should measure the noise level and calculate the load of the forward channel.

\section{REFERENCES}

[1] Chih-Lin I, Charles A. Webb III, Howard C. Huang, Stephan ten Brink, Sanjiv Nanda and Richard D. Gitlin, "IS-95 Enhancements for Multimedia Services," Bell Labs Technical Journal, vol. 1, no. 2, pp. 60-87, Winter, 1996.

[2] D. N. Knisely, S. Kumar, S. Laha and S. Nanda, "Evolution of Wireless Data Services: IS-95 to cdma2000," IEEE Commun. Magazine, pp. 140-149, October, 1998.

[3] Chi-Lin and S. Nanda, "Load and Interference Based Demand Assignment (LIDA) for Integrated Services in CDMA Wireless Systems," Proceedings of the IEEE GLOBECOM Conference, pp. 235-241, London, England, 1996.

[4] R. P. Ejzak, D. N. Knisely, S. Kumar, S. Laha and
S. Nanda, "BALI: A Solution for High-Speed CDMA Data," Bell Labs Tech. J., vol. 2, no. 3, pp. 134-151, Summer 1997. 